\title{
SOLAR IRRADIANCE VARIATIONS: THEORY
}

\author{
H.C. SPRUIT \\ Max-Planck-Institut für Astrophysik \\ Postfach 1523 \\ D-85740 Garching, Germany
}

\section{Introduction}

The following is a somewhat condensed version of discussions previously given elsewhere (Spruit, 1991, 1992). Some new developments not discussed there are presented in sections 4 and 5 .

Since the observed irradiance variations are so clearly associated with manifestations of the solar magnetic field, I focus here on magnetic causes. Much of the physics of irradiance variations, however, is governed by the thermal response of the convective envelope and this response is similar for other possible causes of irradiance variations.

Conceptually, one can separate the thermal effects of magnetic fields into two or three types:

1. 'Sources and sinks'. The generation of a magnetic field involves the conversion of energy of motion into magnetic energy. Since the motions in the solar envelope are thermally driven, this ultimately means conversion of thermal into magnetic energy: building up a magnetic field produces a thermal sink somewhere. The opposite happens when the field decays: magnetic energy is converted into heat. These thermal effects exist only during changes in the magnetic energy content of the envelope.

2. Changes in thermal transport coefficients. Magnetic fields interfere with convection, causing a reduction in the efficiency of heat transport in the envelope. In contrast to (1), these changes last as long as the magnetic field itself is present.

Both these sources and sinks and changes in the transport coefficient cause thermal perturbations, varying with magnetic activity, which propagate through the envelope and cause variations in surface energy flux. Related to the second class of perturbations are:

3. The effects of magnetic fields at the surface of the star. Sunspots, being dark, radiate less than the surrounding photosphere, while the small elements that make up plages and the network have an excess emission. In addition, it is conceivable that the magnetic elements have an indirect effect by modifying the convective flow in their surroundings slightly (for which there is some observational evidence, see section 4). 


\section{Time scales}

The response of the Sun to thermal perturbations is not governed by a single time scale, but by a wide range of time scales. The longest of these is the thermal time scale of the Sun as a whole, called the Kelvin-Helmholtz time scale, about $10^{7}$ years. If the central heat source of the Sun were switched off, the internal structure and the luminosity would start to change on this time scale. More generally, we can define the thermal time $\tau_{\mathrm{t}}$ scale as a function of depth:

$$
\tau_{\mathrm{t}} \equiv U(z) / L(z) \approx \frac{1}{L} \int_{R-z}^{R} 4 \pi r^{2} u \mathrm{~d} r
$$

where $L$ is the luminosity at depth $z, U$ the thermal energy of the envelope down to a depth $z$, and $u$ the thermal energy per unit volume, approximately (for an ideal gas of constant $\gamma$ ) given by $u=P /(\gamma-1)$. This is the time scale on which the structure of the envelope, and the observed luminosity, would start changing when the heat flux in the star were interrupted, by some magical means, at depth $z$. Some rough values for this quantity are $\tau_{\mathrm{t}} \sim 10^{5} \mathrm{yr}$ at $z=210^{5} \mathrm{~km}$ (depth of the convection zone), $10 \mathrm{yr}$ at $20000 \mathrm{~km}$ (the size of a supergranule), $10 \mathrm{hrs}$ at $2000 \mathrm{~km}$ (size of a granule). This shows that the thermal time scale is a very sensitive function of depth in the Sun. As a result, the thermal response of the Sun also depends critically on the location of the disturbance. A fairly good approximation to the stratification of the convective envelope of the Sun is a polytrope of index $n=2$ (this is better than the standard 'convective' value $n=1.5$ because $\gamma<5 / 3$ due to partial ionization). Hence the gas pressure and thermal time scale vary roughly as $P \sim z^{3}, \tau_{\mathrm{t}} \sim z^{4}$, where the depth $z$ is counted from a level 3 scale heights above the surface $\tau=1$.

A second kind of time scale involved in thermal readjustments is the diffusive time scale. In the mixing length approximation, transport processes in the convection zone can be computed with a turbulent diffision coefficient $\kappa_{\mathrm{t}} \approx \frac{1}{3} l_{\mathrm{c}} v_{\mathrm{c}}$ where $l_{\mathrm{c}}$ and $v_{\mathrm{c}}$ are the convective length scale and velocity. In the solar convective envelope, this quantity varies only weakly, at a value of the order $10^{13} \mathrm{~cm}^{2} / \mathrm{s}$. Thermal inhomogeneities (more precisely: entropy inhomogeneities) of length scale $d$ are smoothed by turbulent diffusion on a time scale

$$
\tau_{\mathrm{d}}=d^{2} / \kappa_{\mathrm{t}}
$$

For $d=210^{5} \mathrm{~km}$ this is about $1 \mathrm{yr}$, for $d=2000 \mathrm{~km}$ about $1 \mathrm{hr}$. Comparing $\tau_{\mathrm{t}}$ and $\tau_{\mathrm{d}}$, we see that they are of similar magnitude close to the surface (to be precise: in the surface boundary layer where convection is not efficient enough to keep the stratification close to adiabatic). In deeper layers, the thermal time scale is much longer than the diffusive time scale, by a factor of up to $10^{5}$.

The two time scales measure different types of thermal adjustment process. These same processes appear in the thermal behavior of, say, a chunk of metal, aluminum say, heated from the inside and suspended in space. The thermal time scale is the time scale on which its temperature adjusts to a change in the heat input, such that the heat radiated from the surface into space balances the heat input again. It is determined by the heat capacity $[U$ in eq. (1)] and the power level $(L)$. The time scale on which different parts of the chunk equilibrate to the same temperature is governed by a different process, namely the thermal conduction (the equivalent of the turbulent diffusion in the Sun). The diffusion (conduction) time scale is much shorter than the thermal time scale, because of the large heat conductivity of Al. In the Sun, it is the 
very large turbulent diffusivity in the bulk of the convection zone that causes the very short diffusive time scale compared with the thermal time scale.

How do these different time scales come into play when the convection zone is thermally perturbed by, say, the storage of energy in a growing magnetic field? Such perturbations can be computed in detail, either by numerical methods (Endal et al.1985, Gilliland, 1988) or more analytically. We can, for example, consider the initial value problem in which a perturbation is allowed to evolve in time by thermal transport in the convection zone. In general this evolution has components on all the time scales of the problem, including the very long thermal time scale. Formal aspects of this problem have been discussed elsewhere (Spruit, 1982ab, 1991, Arendt 1992). In the following, the basic conclusions of these analyses are summarized.

\section{Expected level of luminosity variations}

For quantitative estimates, the strength of the field and its filling factor in the convection zone have to be specified. Assume that we have a layer of field with strength of the order of $10000 \mathrm{G}$ (equipartition with the convective flows as estimated by a mixing length model), one scale height deep, near the base of the convection zone (where most of the magnetic flux is probably located). I summarize here some results, dicsussed in greater detail elsewhere (Spruit 1991).

\subsection{SOURCES-AND-SINKS}

If the energy needed to build up this field during one half of the solar cycle is taken out of the thermal energy near the base of the convection zone, the calculations show that a surface luminosity variation of only $10^{-7}$ results. This is due to the very large heat capacity of the lower convection zone. The effect is stronger if the source of the magnetic field is assumed to be closer to the surface, but is still much smaller than the observed effect. Recent models for the emergence of magnetic flux from the base of the convection zone (D'Silva and Choudhuri 1993, D'Silva and Howard 1993, Caligari et al. 1995) indicate that the actual field strength at the base of the convection zone is probably about 10 times higher than the equipartition estimate. The magnetic energy per unit of magnetic flux is then also 10 times higher, but this still does not lead to a significant luminosity effect.

\subsection{SHADOWS}

If magnetic fields interfere with convection, a thermal perturbation develops as well. This effect depends crucially on the 'covering factor'. If a reduction of convective efficiency is assumed that uniformly covers a horizontal surface at some depth $z$, magnetic fields comparable to equipartition with convective flows can have stronger effects than the source-and-sink perturbations (Gilliland, 1988), though a measurable effect is predicted only if the field is located close below the surface. The effect is reduced, however, if there are 'holes' in this cover. This is because the turbulent heat conductivity in the convection zone is so high that the heat flux is easily 'shunted' past blocking objects below the surface (Spruit, 1977). 


\subsection{SURFACE EFFECTS}

By far the most effective way in which a magnetic field influences the irradiance is by its effect on the net surface emissivity. The reduced emission from a sunspot area shows up directly in the irradiance records as a dip tracking the passage of the area across the disk. The only complication is that one might expect that part of this reduction could be compensated by a brightening elsewhere, for example in the form of a 'bright ring' surrounding the spot. Evidence of such bright rings is absent for most spots that have been studied for this effect. Any brightening in the surroundings can usually be attributed to facular emission, which is present at the same levels in active regions with or without sunspots. The absence of such bright rings is understood in terms of a turbulent diffusion model for the heat flux in the convection zone (Spruit, 1977, 1982b, Foukal et al. 1983). The 'blocked heat flux', for the most part, does not reappear elsewhere on the surface, but stays inside the convection zone, being stored/released on the very long thermal time scale of the convective envelope. This conclusion holds, in the diffusion model, as long as the blocking effect of the spot extends to a depth of at least $1000 \mathrm{~km}$, a mild requirement given that the observed Wilson depression of the umbra of a spot is already of the order of $500 \mathrm{~km}$.

Changes in heat flux through modified granulation will affect the convective envelope in the same way as the excess emission from the small scale magnetic field, since it is also an effective change of the emissivity of the solar surface. In particular, one does not expect these changes to be 'compensated' by opposite changes elsewhere on the surface (except, as before, on the $10^{5} \mathrm{yr}$ thermal time scale of the envelope).

\section{4. 'Magnetically modified' granulation}

In 3.3 above, I have focused on the direct effects of magnetic fields on the net emissivity of the solar surface. More indirect effects may play a role as well. The shape of granules appears to be different in magnetic regions (Macris and Roesch 1983, Muller and Roudier 1984, Muller 1986, Title et al. 1992). They are smaller, more irregular, and the measured flow speeds are lower. These effects are present in addition to the magnetic elements themselves, which change the appearance of granulation by filling in the intergranular lanes. The changes may be the result of the geometric constraints the magnetic tubes put on the convective flow outside them.

Since the flow appears to be different, it would seem possible that the heat flux it carries is also different. This might contribute to the observed solar cycle variations of irradiance (Muller 1986, Kuhn and Libbrecht 1988). Limits on this contribution can be put by observations of the colors of the solar cycle variation signal. Solanki (1997) shows that the variation as seen in the near UV follows the behavior expected from the small magnetic elements themselves rather than the weak color signal expected from a small variation in the surface temperature over a larger area, but he does not quote quantitative limits. In order to contribute in the right sense to the solar cycle variation, the constraints imposed on the flow by the magnetic flux elements would have to lead to an increase in the heat flux carried by granulation. Direct measurements of a temperature change in granulation in magnetic regions are probably difficult, since the effect would be small and hard to separate from the enhanced emission from the magnetic elements. Numerical simulations (Nordlund, private communication) indicate, however, that an effect of the right sign may be present. 


\section{Improving on the turbulent diffusion picture}

In the results quoted a diffusion model for convection was used. It assumes that convection can be modeled by a turbulent viscosity for momentum and a turbulent diffusivity for the transport of heat. While this was a simple and somewhat justifiable model of convection in the absence of detailed knowledge of the convective flows in a stellar envelope, we now know that it does not represent stellar convective envelopes very well. Numerical simulations (Nordlund 1982, 1985ab, 1986, 1990, Nordlund and Dravins 1990, Nordlund and Stein 1990, 1991, 1996, Stein and Nordlund 1989, 1991, Steffen et al. 1989, Steffen 1993) show an extremely nonlocal picture. They show that convective flows are driven almost exclusively by cooling at the surface, with narrow fast moving downdrafts between slow almost isentropic upflows. For detailed descriptions of these results, see the references given (cf also Spruit 1997). In the present context, the most important property is that the convective flow at all depths is driven by cooling at the surface rather than by a local overturning process. The material in the cool downdrafts survives to large depths below the surface with little mixing into the upflows.

\subsection{SPOT BLOCKING}

Given this extremely nonlocal picture, it is appropriate to ask how the sunspot blocking problem can be explained without appealing to a turbulent diffusion model.

Below the spot (modeled as a region of reduced heat transport efficiency extending to some depth below the surface) the upflows have exactly the same temperature as upflows in the unspotted surroundings at the same level, namely that given by the entropy at the base of the upflows. In this sense, there is no 'pile up of heat below the spot'. Because of the reduced heat loss at the surface, however, the downflows below the spot will be less vigorous. The unspotted surface notices nothing of the spot's presence (except for an extremely narrow ring where lateral radiative exchange takes place, and except for the presence of a moat flow, see below). It continues to cool upwellings into downdrafts as before, since the entropy in the upflows has not changed. Thus, we expect again that bright rings will be absent around spots, but the reason is even simpler than in the diffusion model. The spot is a region at the surface where less heat is radiated away, and this is now independent of the depth of the spot below the surface (in contrast to the diffusion model, where the spot has to extend to a minimum depth of $1000 \mathrm{~km}$ for the explanation to work).

Still, one may wonder what happens to the amount of heat generated in the solar interior that now fails to be emitted at the surface. This part of the problem is the same as in the diffusion model: the imbalance causes a secular increase of the entropy in the entire convection zone until a new thermal equilibrium is reached. Because of the very long thermal time scale of the convection zone $\left(10^{5} \mathrm{yr}\right)$, the effect is negligible on observable time scales. In a steady state, when the average number of spots does not change, the convection zone does not heat up, because its mean temperature is higher than it would be without spots (Spruit and Weiss, 1986). Episodes of larger than average spot coverage cause heating, those of less than average spot coverage cause cooling on this time scale. 


\subsection{MOAT CIRCULATION}

In the diffusion model, the horizontal flow away from a spot observed as the 'moat' has a simple explanation, since the convection zone is hotter than average below the spot in this model, causing an upflow by thermal buoyancy. In our new view of the convection zone, the explanation does not work any more in this simple form, since there is no upward buoyancy anywhere. A circulation still results, however, due to the buoyancy associated with a reduced density below the spot. Downflows are cooler and denser than the surrounding upflows, so that the mean density on a horizontal surface is greater than the density in the upflowing fluid. Below the spot this effect is less strong than outside the spot, since less cooling has taken place at the surface. This difference in mean buoyancy of the fluid below the spotted and unspotted areas drives a circulation as before. The difference in explanation may sound a bit pedantic, since one could also have said that the mean temperature below the spot is higher than outside because of the weaker downflows. The new explanation is more satisfactory since it appeals more directly to the physical cause of the circulation, namely an imbalance in the fluid density. More importantly, this explanation shows that the moat circulation does not carry extra heat to the surface: on a given horizontal level below the surface the mean temperature is higher in the circulation, but this increase is entirely caused by fluid which is moving downward, and therefore not visible at the surface, rather than upward as it is in the diffusion picture! This difference again is due to the extremely nonlocal nature of convection in stellar envelopes.

\subsection{THERMAL SHADOWS}

The thermal shodow expected from a blocking object below the surface (Spruit 1977, Goode and Kuhn, 1990), which is a small effect already in the turbulent diffusion model, is likely to be even smaller in the new picture of stellar envelope convection. Since all upward flowing gas has nearly the same entropy, the only effect of a blocking object is to temporarily halt the downward motion of gas cooled at the surface. As in the case of the moat circulation discussed above, this has no consequence for the surface temperature above the blocking object, since only a small fraction of the downward moving gas is carried back up to the surface. As before, this is a consequence of the non-local nature of the convective flow.

\section{Acknowledgement}

It is a pleasure to thank Alan Title for a discussion on the effects of surface magnetic fields on granulation, which led to the inclusion of section 4.

\section{References}

Arendt, S., 1992, Astrophys. J 389, 421

Caligari, P., Moreno-Insertis, F., Schuessler, M., 1995, Astrophys., J. 441, 886

Chiang, W. H. \& Foukal, P. V., 1984, Solar Phys. 97, 9

D'Silva, S. \& Choudhuri, A. R., 1993 Astron. Astrophys. 272, 621

D'Silva \& Howard, R. A. 1993, Solar Phys. 148, 1

Endal, A. S., Sofia, S. \& Twigg L. W., 1985, Astrophys. J. 290, 748

Foukal, P. V., Fowler, P., \& Livshits, M., 1983, Astrophys. J. 267, 863 
Gilliland, R. L., 1988, in Solar radiative output variation, P. Foukal, ed., Cambridge Research \& Instrumentation Inc., 21 Erie st. Cambridge, MA 02139, p239

Goode, P.R., Kuhn, J.R., 1990, Astrophys. J. 356, 310

Kuhn, J.R., Libbrecht, K.G., Dicke, R.H., 1988, Science 242, 908

Muller, R., 1986 Solar Phys., 119, 229

Muller, R., Roudier, T., 1984, in The Hydromagnetics of the Sun, ESA SP xx, p. 51

Macris, C.J., and Roesch, J., 1983, Comptes Rendus, ser II, 296, 265

Nordlund, A., 1982, Astron. Astrophys. 107, 1

Nordlund, $\AA ., 1985 \mathrm{a}$, in Small scale dynamical processes in quiet stallar atmospheres ed. W. Keil, Sacramento Peak Observatory, Sunspot, NM 88349, USA

Nordlund, $\AA ., 1985 \mathrm{~b}$, in Progress in stellar spectral line formation theory, eds. J.E. Beckman and L. Crivellari (NATO ASI series 152), Reidel, Dordrecht, p. 215

Nordlund, A., 1986, Solar Phys., 100, 209

Nordlund, A., 1991, in Stellar atmopheres: Beyond classical models, eds. L. Crivellari, I. Hubeny and D.G. Hummer (NATO ASI series 341), Kluwer, Dordrecht, p. 61

Nordlund, A. \& Dravins, D., 1990, Astron. Astrophys. 228, 155

Nordlund, A. \& Stein, R.F., 1990, Comp. Phys. Comm. 59, 119

Nordlund, A. \& Stein, R.F., 1991, in Stellar atmopheres: beyond classical models, eds. L. Crivellari, I. Hubeny and D.G. Hummer (NATO ASI series 341), Kluwer, Dordrecht, p. 263

Nordlund, Å. \& Stein, R.F., 1996, in Proceedings of the $32^{n} d$ Liège Int. Astrophys. Colloquium 'Stellar Evolution: What should be done', eds. A. Noels et al. p.75

Solanki, S., 1997, submitted to Astron. Astrophys.

Spruit, H.C., 1977, Solar Phys. 55, 3

Spruit, H.C. 1982a, Astron. Astrophys. 108, 348

Spruit, H.C. 1982b, Astron. Astrophys. 108, 356

Spruit, H.C. 1991, in The Sun in Time, eds. C. Sonett, M. Giampapa \& M.S. Matthews, University of Arizona Press, Tucson, p118

Spruit, H.C., 1992, in Sunspots: Theory and Observations, eds. J.H. Thomas and N.O. Weiss, Cambridge: CUP, p.163

Spruit, H.C., 1997, Mem. Soc. Astron. It., in press

Spruit, H.C. \& Weiss, A., 1986, Astron. Astrophys. 166, 167

Steffen, M., Ludwig, H.-G., Krüss, A.: 1989 Astron. Astrophys. 213, 317

Steffen, M.: 1993, in Inside the stars (IAU Coll 137), eds. W. Weiss \& A. Baglin, Astron. Soc. Pac. Conference series 40, p300

Stein, R.F. \& Nordlund, §, 1989, Astrophys. J. 342, L95

Stein, R.F. \& Nordlund, $\AA, 1991$, in Challenges to Theories of the Structure of ModerateMass Stars, eds. D.O. Gough and J. Toomre, Lecture Notes in Physics 388, Springer, Berlin, p195

Title, A.M., Topka K.P., Tarbell T.D., Schmidt, W., Balke C., Scharmer G., 1992, Astrophys. J. 393, 782 